\title{
Association between Recipient IL-15 Genetic Variant and the Prognosis of HBV-Related Hepatocellular Carcinoma after Liver Transplantation
}

\author{
Tao Zhang, ${ }^{1}$ Yuan Liu, ${ }^{1}$ Xu Peng, ${ }^{2}$ Junwei Fan, ${ }^{1}$ and Zhihai Peng ${ }^{1}$ \\ ${ }^{1}$ Department of Hepatobiliary Pancreatic Surgery, Shanghai General Hospital, School of Medicine, Shanghai Jiao Tong University, \\ Shanghai, China \\ ${ }^{2}$ Department of General Surgery, Hainan Cancer Hospital, Haikou, China
}

Correspondence should be addressed to Junwei Fan; fanjw880301@163.com and Zhihai Peng; pengzh1958@163.com

Received 2 June 2017; Revised 20 August 2017; Accepted 14 September 2017; Published 15 October 2017

Academic Editor: Yi-Chia Huang

Copyright (c) 2017 Tao Zhang et al. This is an open access article distributed under the Creative Commons Attribution License, which permits unrestricted use, distribution, and reproduction in any medium, provided the original work is properly cited.

\begin{abstract}
Objective. To investigate the association of donor and recipient IL-15 genetic variants with HCC recurrence and prognosis after LT. Methods. A total of 112 liver transplant patients with HBV-related HCC were enrolled. IL-15 rs10519613 and rs13122930 were genotyped in donors and recipients. Results. Recipient IL-15 rs10519613 polymorphism was found to be significantly related to HCC recurrence after LT. In multivariate analysis, tumor thrombus, UCSF criteria, and recipient IL-15 rs10519613 genotypes were independent predictive factors of HCC recurrence after LT. Kaplan-Meier survival analysis demonstrated that patients with recipient IL-15 rs10519613 CA/AA genotypes had a decreased disease-free survival and overall survival than those with the CC genotype. Recipient IL-15 rs10519613 genetic variant could improve survival prediction when combined with the UCSF criteria. Furthermore, Cox proportional hazard regression analysis revealed that tumor size $(p=0.012, p=0.623)$, tumor thrombus $(p=0.011, p=0.015)$, UCSF criteria $(p=0.471, p=0.013)$, and recipient IL-15 rs10519613 genotype $(p=0.039$, $p=0.008$ ) were independent factors of predicting DFS and OS. Conclusions. Recipient IL-15 rs10519613 polymorphism was associated with HCC recurrence after LT and might be a potential genetic marker for the clinical outcome of HCC patients treated with LT.
\end{abstract}

\section{Introduction}

Hepatocellular carcinoma (HCC) is one of the most common malignancies and the third leading cause of cancer-related mortality worldwide $[1,2]$. Liver transplantation provides an effective therapeutic option for HCC and the underlying liver cirrhosis. However, HCC recurrence after LT is a serious complication and negatively affects patient survival.

Clinical parameters such as alpha-fetoprotein (AFP), histologic grade, tumor numbers, tumor size, microvascular invasion, tumor thrombus, tumor stage (TNM), and the selection criteria for LT (Milan or UCSF criteria) have been reported as risk factors for HCC recurrence and prognosis after LT [3-8]. Although several criteria are available for transplant patient selection, they have limitations for determining clinical outcome. Molecular markers and genetic variants could provide supplemental and useful information for predicting the clinical outcome and improve the selection of patients for adjuvant therapies after LT in addition to the clinical factors $[9,10]$. As such, it is a challenge to identify patients who are with a greater risk for HCC recurrence after $\mathrm{LT}$ and potential biomarkers for prognosis prediction.

IL-15 (interleukin 15) encodes the cytokine that is a four- $\alpha$-helix-bundle cytokine and plays a pivotal role in the proliferation and survival of T lymphocytes, NK cells, NKT cells, and different subsets of innate lymphoid cells. More specifically, IL-15 increases the cytotoxicity of $\mathrm{CD}^{+} \mathrm{T}$ cells and is essential for NK cell activation and thus is involved 
TABLE 1: Demographic data for recurrence and nonrecurrence groups.

\begin{tabular}{|c|c|c|c|}
\hline & Recurrence group $(n=38)$ & Nonrecurrence group $(n=74)$ & $p$ value \\
\hline Recipient age (yr) & $48.47 \pm 7.95$ & $50.93 \pm 8.82$ & 0.152 \\
\hline Recipient male/female $(n)$ & $35(92.1) / 3(7.9)$ & $67(90.5) / 7(9.5)$ & 0.783 \\
\hline Cirrhosis yes/no $(n)$ & $30(78.9) / 8(21.1)$ & $65(87.8) / 9(12.2)$ & 0.214 \\
\hline Histologic grade $(n)$ & & & 0.203 \\
\hline Differentiated & $28(73.7)$ & $62(83.8)$ & \\
\hline Undifferentiated & $10(26.3)$ & $12(16.2)$ & \\
\hline Tumor size $(n)$ & & & $<0.001$ \\
\hline$<5 \mathrm{~cm}$ & $16(42.1)$ & $57(77.0)$ & \\
\hline$\geq 5 \mathrm{~cm}$ & $22(57.9)$ & $17(23.0)$ & \\
\hline Multinodular yes/no $(n)$ & $19(50.0) / 19(50.0)$ & $28(37.8) / 46(62.2)$ & 0.217 \\
\hline Tumor thrombus $(n)$ & & & $<0.001$ \\
\hline Yes & $24(63.2)$ & $19(25.7)$ & \\
\hline No & $14(37.8)$ & $55(74.3)$ & \\
\hline Serum AFP level at LT $(n)$ & & & 0.489 \\
\hline$<400 \mathrm{~cm}$ & $27(71.1)$ & $57(77.0)$ & \\
\hline$\geq 400 \mathrm{~cm}$ & $11(28.9)$ & $17(23.0)$ & \\
\hline Tumor stage $(n)$ & & & 0.003 \\
\hline I-II & $22(57.9)$ & $62(83.8)$ & \\
\hline III & $16(42.1)$ & $12(16.2)$ & \\
\hline UCSF criteria in/out $(n)$ & $18(47.7) / 20(52.3)$ & $57(77.0) / 17(23.0)$ & 0.002 \\
\hline
\end{tabular}

in Th1-type immune response, including antitumor response [11-16]. Accumulated evidences have shown that IL-15 was significantly associated with a decreased incidence of tumor recurrence and a prolonged overall survival, including HCC $[17,18]$. It is well known that, besides the inflammatory response, some other factors including the genetic variants also play an important role in the expression and activity of cytokines [19]. Several studies have revealed that the genetic variants of a cytokine gene were associated with HCC and HCC recurrence $[10,20,21]$.

However, the influence of the IL-15 gene polymorphisms on the prognosis of HCC after LT has not been investigated. In this study, we selected two potentially significant SNPs of IL-15 and aimed to investigate whether donor and recipient IL-15 polymorphisms were associated with HCC recurrence and prognosis after LT in a Han Chinese population.

\section{Patients and Methods}

2.1. Patients. A total of 112 hepatitis B virus- (HBV-) related HCC patients who underwent orthotopic liver transplantation between July 2007 and June 2014 at Shanghai Jiao Tong University Affiliated Shanghai General Hospital, China, were enrolled. There were 102 males and 10 females with a mean age of $50.1 \pm 8.6$ years (range: $21-67$ years). The main clinicopathological characteristics of the study population are summarized in Table 1. All patients were routinely followed up at the posttransplant clinic according to our previous study [22], and the mean follow-up time was $38.3 \pm 27.9$ months (range: 1-105 months).
2.2. Ethics Statement. Liver grafts were obtained from donation after cardiac death (DCD) as well as from living related donors. In our center, almost all liver grafts were obtained from DCD; only one liver graft was obtained from LD in the study. Informed consent was obtained from all subjects. The research was approved by the Ethics Committee of Shanghai Jiao Tong University. The methods were carried out in accordance with the Declaration of Helsinki and its later amendments or comparable ethical standards.

2.3. Data Collection. The following data were collected before LT: patient age, gender, underlying liver disease (HBV), and preoperative AFP level $(<400 \mathrm{ng} / \mathrm{ml}$ or $\geq 400 \mathrm{ng} / \mathrm{ml})$. After LT, tumor size and number, histologic grade (differentiated: well/moderate; undifferentiated: poor), tumor thrombus, pathological TNM staging, cirrhotic background, diseasefree survival (DFS), and overall survival (OS) were recorded. The diagnosis of HCC was confirmed by two pathologists. Each tumor in the explant liver was reevaluated to give a judgment according to the UCSF criteria (one tumor of $\leq 6.5 \mathrm{~cm}$ or a maximum of three tumor nodules each of $\leq 4.5 \mathrm{~cm}$ and the sum of tumor diameters of $\leq 8 \mathrm{~cm}$ ) on the basis of pathological data [23].

2.4. Genomic DNA Isolation and Genotyping. The two potentially significant SNPs (rs10519613 and rs13122930) of IL-15 were selected by our exon array, the International HapMap Project database, and the dbSNP database (a minor allele frequency $>0.05$ in Asian populations). Genomic DNA was extracted from the donor and recipient liver tissue (which had been previously stored at $-80^{\circ} \mathrm{C}$ ) using an AllPrep 
DNA/RNA Mini Kit (Qiagen, Hilden, Germany). Genotyping of IL-15 rs10519613 and rs13122930 was conducted using the Sequenom MassARRAY SNP genotyping platform (Sequenom, San Diego, CA, USA) [24]. The protocols included polymerase chain reaction amplification, shrimp alkaline phosphatase treatment, single-base extension reaction, resin cleanup, nanodispensing on SpectroCHIP, and data acquisition. To confirm the genotyping results, $>10 \%$ of the samples were randomly selected and regenotyped with $100 \%$ concordance.

2.5. Statistical Analysis. Statistical analysis was performed using SPSS version 19.0 (IBM Corp., Armonk, NY, USA) and GraphPad Prism Version 5.00 software (GraphPad Inc., La Jolla, CA, USA). The Hardy-Weinberg equilibrium and the allele frequency were analyzed using SHEsis software [25]. Quantitative variable data were compared using Student's $t$-test, and categorical variables were analyzed by the chi-square test or Fisher's exact test. All variables were evaluated by univariate logistic regression analysis. Variables with statistical significance in univariate analysis were included in the multivariate analysis. Disease-free survival (DFS) and overall survival (OS) between different groups were performed with Kaplan-Meier survival curves and compared using the log-rank test, and variables with significance in univariate Cox proportional hazard regressions were performed in the multivariate analysis. Two-tailed $p$ values of less than 0.05 were considered statistically significant.

\section{Results}

3.1. Clinicopathological Characteristics of LT Patients. The overall incidence of HCC recurrence after LT was $33.9 \%$ $(38 / 112)$ in this study population. The clinicopathological characteristics in the recurrence and nonrecurrence groups are shown in Table 1. Tumor size $(p<0.001)$, tumor thrombus $(p<0.001)$, TMN stage $(p=0.003)$, and UCSF criteria $(p=0.002)$ were significantly associated with HCC recurrence after LT. However, no significant difference was observed between the two groups concerning other clinicopathological characteristics, including the recipient age, gender, cirrhotic background, histologic grade, tumor number, and serum AFP level.

\subsection{Effect of IL-15 rs10519613 and rs13122930 Genotypes on} HCC Recurrence. Genotype distribution of donor and recipient IL-15 rs10519613 and rs13122930 and the effect of the two SNPs on HCC recurrence after LT are shown in Table 2. The observed SNPs were in accordance with the Hardy-Weinberg equilibrium $(p>0.05)$. No significant linkage disequilibrium between the two SNPs was observed. The incidence of HCC recurrence was significantly higher for patients with recipient IL-15 rs10519613 CA/AA genotypes than for those with the CC genotype $(p<0.001)$, and patients with the recipient IL-15 rs10519613 A allele had higher HCC recurrence than those with the $\mathrm{C}$ allele $(p<0.001)$. However, donor IL-15 rs10519613 and donor and recipient IL-15 rs13122930 polymorphisms were not significantly associated with HCC recurrence in our study.
3.3. Univariate and Multivariate Analyses for Risk Factors for HCC Recurrence. Univariate and multivariate analyses were used to determine the association between the risk factors and HCC recurrence (Tables 3 and 4). In univariate analysis, tumor size $(p<0.001)$, tumor thrombus $(p<0.001)$, TMN stage $(p=0.004)$, UCSF criteria $(p=0.002)$, and recipient IL15 rs10519613 genotypes (CA/AA versus CC, $p=0.001$ ) were significantly associated with an increased risk of HCC recurrence. All significant factors $(p<0.05)$ identified by univariate analysis were included in a multivariate analysis. Tumor thrombus (OR $=3.591,95 \% \mathrm{CI}: 1.438-8.971, p=0.006), \mathrm{UCSF}$ criteria $(\mathrm{OR}=3.922,95 \% \mathrm{CI}: 1.515-10.152, p=0.005)$, and recipient IL-15 rs10519613 genotypes (CA/AA versus CC; $\mathrm{OR}=5.143,95 \% \mathrm{CI}: 1.636-16.168, p=0.005)$ were independent predictive factors of HCC recurrence.

3.4. Survival Analysis. Univariate Cox regression analysis revealed that histologic grade, tumor size, tumor thrombus, TMN stage, UCSF criteria, recipient IL-15 rs10519613 genotypes (CA/AA versus CC) were significantly associated with DFS and OS (Table 5). A Kaplan-Meier plot of patient survival is shown in Figures 1(a) and 1(b). The group of patients with recipient IL-15 rs10519613 CA/AA genotypes had a reduced DFS (log-rank; $p=0.0038$; Figure $1(\mathrm{a}))$ and OS (log-rank; $p=0.0003$; Figure $1(\mathrm{~b}))$ than the group with the recipient IL-15 rs10519613 CC genotype.

The results of multivariate analysis showed that tumor size $(\mathrm{OR}=2.213,95 \% \mathrm{CI}: 1.188-4.122, p=0.012)$, tumor thrombus $(\mathrm{OR}=2.385,95 \% \mathrm{CI}: 1.224-4.647, p=0.011)$, and recipient IL-15 rs10519613 genotypes (CA/AA versus $\mathrm{CC}$; $\mathrm{OR}=2.214$, 95\% CI: $1.041-4.708, p=0.039)$ were independent factors of predicting DFS, whereas tumor thrombus (OR $=2.302,95 \%$ CI: $1.173-4.517, p=0.015)$, UCSF criteria $(\mathrm{OR}=2.209,95 \% \mathrm{CI}: 1.181-4.131, p=0.013)$, and recipient IL-15 rs10519613 genotypes (CA/AA versus CC; $\mathrm{OR}=3.152$, 95\% CI: $1.358-7.315, p=0.008)$ were independent factors of predicting OS (Table 6).

\subsection{Improved Survival Prediction after Integrating Recipient} IL-15 rs10519613 Genetic Factor into the UCSF Criteria. To investigate whether recipient IL-15 rs10519613 genetic variant could improve survival prediction when combined with the UCSF criteria, survival differences between the patients with tumors within or beyond the UCSF criteria stratified by recipient IL-15 rs10519613 genetic variant were analyzed. For patients who met the UCSF criteria, recipient IL-15 rs10519613 genotypes (CA/AA versus CC) could independently predict DFS $(p=0.0343$, Figure $2(\mathrm{a}))$ and OS $(p=0.0100$, Figure $2(b))$. And for patients who did not meet the UCSF criteria, significant differences in DFS $(p=0.0218$, Figure $3(\mathrm{a}))$ and OS $(p=0.0063$, Figure $3(\mathrm{~b}))$ between the two genotype groups were also observed.

\section{Discussion}

Although some researches have reported the association of IL-15 with the clinical outcome of HCC, studies of the genetic variants in the IL-15 gene on HCC recurrence and prognosis after LT are few. In this study, the overall 
TABLE 2: Comparison of genotype and allele distribution between recurrence and nonrecurrence groups.

\begin{tabular}{|c|c|c|c|c|c|}
\hline SNP (allele) & \multicolumn{3}{|c|}{ Genotype distribution $(n)$} & $p$ value & HWE $p$ value \\
\hline \multicolumn{6}{|l|}{ Donor } \\
\hline rs10519613 & $\mathrm{CC}$ & 8 & 27 & & 0.219 \\
\hline \multirow{6}{*}{$(\mathrm{C} / \mathrm{A})$} & $\mathrm{CA}$ & 22 & 36 & 0.132 & \\
\hline & AA & 8 & 11 & 0.139 & \\
\hline & $\mathrm{CC}$ & 8 & 27 & & \\
\hline & CA/AA & 30 & 47 & 0.095 & \\
\hline & $\mathrm{C}$ & 38 & 90 & & \\
\hline & $\mathrm{A}$ & 38 & 58 & 0.122 & \\
\hline rs13122930 & TT & 11 & 13 & & 0.441 \\
\hline \multirow{6}{*}{$(\mathrm{T} / \mathrm{C})$} & TC & 19 & 41 & 0.221 & \\
\hline & $\mathrm{CC}$ & 8 & 20 & 0.198 & \\
\hline & $\mathrm{TT}$ & 11 & 13 & & \\
\hline & $\mathrm{TC} / \mathrm{CC}$ & 27 & 61 & 0.165 & \\
\hline & $\mathrm{C}$ & 41 & 67 & & \\
\hline & A & 35 & 81 & 0.218 & \\
\hline \multicolumn{6}{|l|}{ Recipient } \\
\hline rs10519613 & $\mathrm{CC}$ & 5 & 35 & & 0.072 \\
\hline \multirow{6}{*}{$(\mathrm{C} / \mathrm{A})$} & $\mathrm{CA}$ & 21 & 28 & 0.002 & \\
\hline & AA & 12 & 11 & 0.001 & \\
\hline & $\mathrm{CC}$ & 5 & 35 & & \\
\hline & CA/AA & 33 & 39 & $<0.001$ & \\
\hline & $\mathrm{C}$ & 31 & 98 & & \\
\hline & A & 45 & 50 & $<0.001$ & \\
\hline rs13122930 & TT & 9 & 13 & & 0.294 \\
\hline \multirow{6}{*}{$(\mathrm{T} / \mathrm{C})$} & TC & 18 & 31 & 0.738 & \\
\hline & $\mathrm{CC}$ & 11 & 30 & 0.252 & \\
\hline & $\mathrm{TT}$ & 9 & 13 & & \\
\hline & $\mathrm{TC} / \mathrm{CC}$ & 29 & 61 & 0.440 & \\
\hline & $\mathrm{T}$ & 36 & 57 & & \\
\hline & $\mathrm{C}$ & 40 & 91 & 0.203 & \\
\hline
\end{tabular}

SNP: single-nucleotide polymorphism; HWE: Hardy-Weinberg equilibrium.

TABLE 3: Univariate logistic regression analysis of risk factors associated with tumor recurrence.

\begin{tabular}{lrr}
\hline Risk factors & $p$ value & Odds ratio (95\% CI) \\
\hline $\begin{array}{l}\text { Tumor size } \\
(0:<5 \mathrm{~cm}, 1: \geq 5 \mathrm{~cm})\end{array}$ & $<0.001$ & $4.610(1.987-10.695)$ \\
$\begin{array}{l}\text { Tumor thrombus } \\
(0=\text { no, } 1=\text { yes })\end{array}$ & $<0.001$ & $4.962(2.141-11.501)$ \\
$\begin{array}{l}\text { Tumor stage }(n) \\
(0=\mathrm{I}-\mathrm{II}, 1=\mathrm{III})\end{array}$ & 0004 \\
$\begin{array}{l}\mathrm{UCSF} \text { criteria }(n) \\
(0=\text { no, } 1=\text { yes })\end{array}$ & $3.758(1.537-9.174)$ \\
$\begin{array}{l}\text { Recipient IL-15 rs } 10519613 \text { genotype } \\
(0=\text { CC, } 1=\text { CA/AA) }\end{array}$ & 0.002 & $3.758(1.615-8.595)$ \\
\hline
\end{tabular}

CI: confidence interval. 
TABLE 4: Multivariate logistic regression analysis of risk factors associated with tumor recurrence.

\begin{tabular}{lrr}
\hline Risk factors & $p$ value & Odds ratio (95\% CI) \\
\hline $\begin{array}{l}\text { Tumor thrombus } \\
(0=\text { no, } 1=\text { yes })\end{array}$ & 0.006 & $3.591(1.438-8.971)$ \\
$\begin{array}{l}\text { UCSF criteria }(n) \\
(0=\text { no, } 1=\text { yes })\end{array}$ & 0.005 & $3.922(1.515-10.152)$ \\
Recipient IL-15 rs10519613 genotype & & \\
$(0=$ CC, $1=$ CA/AA) & 0.005 & $5.143(1.636-16.168)$ \\
\hline
\end{tabular}

CI: confidence interval.

TABLE 5: Univariate analysis of prognostic factors associated with DFS and OS.

\begin{tabular}{|c|c|c|c|c|}
\hline Prognostic factors & $\begin{array}{c}\text { DFS (months) } \\
\text { Odds ratio }(95 \% \mathrm{CI})\end{array}$ & $p$ value & $\begin{array}{c}\text { OS (months) } \\
\text { Odds ratio (95\% CI) }\end{array}$ & $p$ value \\
\hline \multicolumn{5}{|l|}{ Histologic grade } \\
\hline$(0=$ differentiated, $1=$ undifferentiated $)$ & $2.093(1.119-3.915)$ & 0.021 & $2.194(1.162-4.139)$ & 0.015 \\
\hline Tumor size $(0:<5 \mathrm{~cm}, 1: \geq 5 \mathrm{~cm})$ & $3.238(1.818-5.768)$ & $<0.001$ & $3.253(1.791-5.910)$ & $<0.001$ \\
\hline Tumor thrombus $(0=$ no, $1=$ yes $)$ & $3.893(2.101-7.015)$ & $<0.001$ & $3.791(2.035-7.060)$ & $<0.001$ \\
\hline Tumor stage $(0=$ stages I-II, $1=$ stage III $)$ & $3.276(1.818-5.904)$ & $<0.001$ & $3.124(1.695-5.756)$ & $<0.001$ \\
\hline UCSF criteria $(0=$ no, $1=$ yes $)$ & $2.769(1.556-4.927)$ & 0.001 & $2.813(1.552-5.097)$ & 0.001 \\
\hline \multicolumn{5}{|l|}{ Donor IL-15 rs10519613 genotype } \\
\hline$(0=\mathrm{CC}, 1=\mathrm{CA} / \mathrm{AA})$ & $2.267(1.096-4.690)$ & 0.027 & $1.778(0.878-3.601)$ & 0.110 \\
\hline \multicolumn{5}{|l|}{ Recipient IL-15 rs10519613 genotype } \\
\hline$(0=\mathrm{CC}, 1=\mathrm{CA} / \mathrm{AA})$ & $3.000(1.447-6.219)$ & 0.003 & $4.005(1.776-9.030)$ & 0.001 \\
\hline
\end{tabular}

DFS: disease-free survival; OS: overall survival; CI: confidence interval.

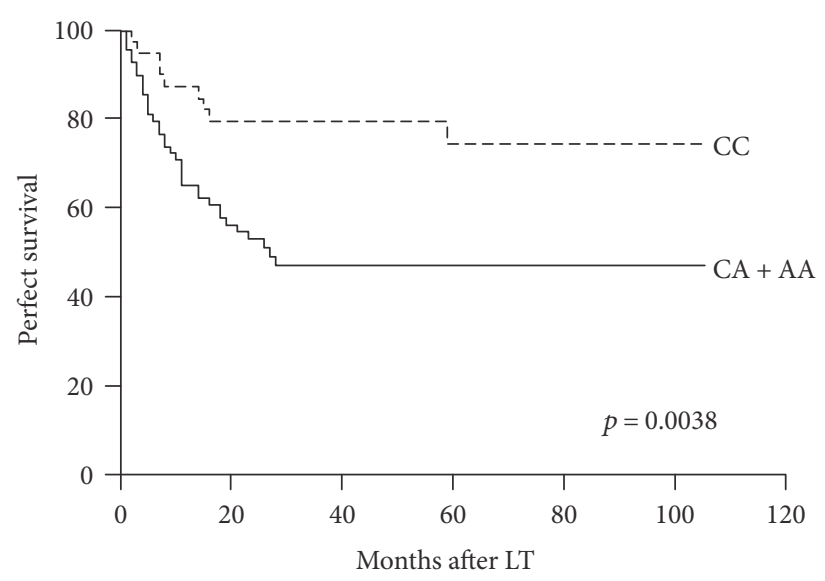

(a)

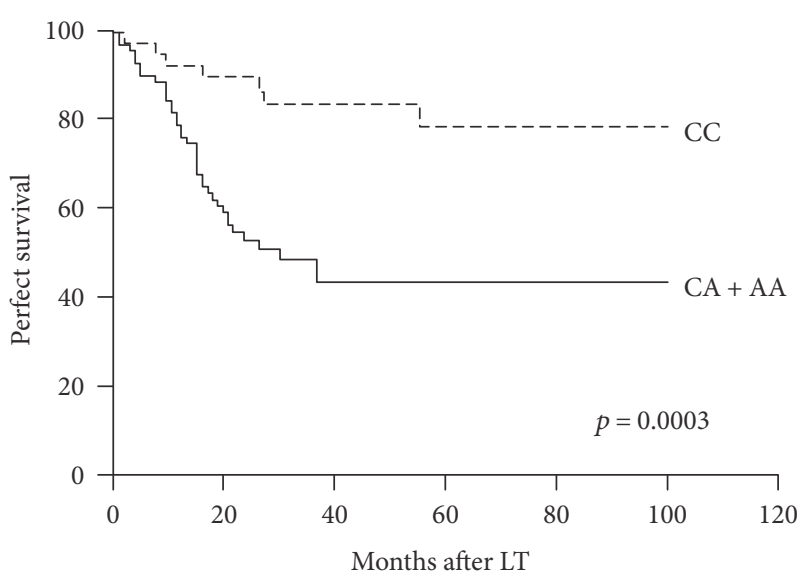

(b)

FIGURE 1: Disease-free survival (a) and overall survival (b) of transplant HCC patients stratified by recipient IL-15 rs10519613 genotypes.

frequency of IL-15 rs10519613 CC, CA, and AA genotypes were $33.5 \%, 47.8 \%, 18.7 \%$, respectively; our data were similar to that of the previous study in other population [26]. Our results revealed that recipient IL-15 rs10519613 polymorphisms (CA/AA versus CC genotype; A versus C allele) were significantly associated with HCC recurrence after LT. In multivariate analysis, recipient IL-15 rs10519613 genotypes (CA/AA versus CC) were independent predictive factors of HCC recurrence after LT. Furthermore, we found that patients with recipient IL-15 rs10519613 CA/AA genotypes had a decreased DFS and OS than patients with the CC genotype in univariate and multivariate Cox proportional hazard regression analyses.

To our knowledge, this is the first study to demonstrate that recipient IL-15 rs10519613 genetic variant influences HCC recurrence and prognosis after LT at the genetic level. IL-15 is a proinflammatory cytokine with similar functions to interleukin 2 (IL-2), including antitumor activity. Given its pivotal role in the proliferation, survival, and activation of $\mathrm{CD}^{+} \mathrm{T}$ cells and NK cells, IL-15 has been identified as an antitumor cytokine in several cancers, including neuroblastoma, HCC, and breast and colorectal cancer [27-31]. 
TABLE 6: Multivariate analysis of prognostic factors associated with DFS and OS.

\begin{tabular}{lccr}
\hline Prognostic factors & $\begin{array}{c}\text { DFS (months) } \\
\text { Odds ratio (95\% CI) }\end{array}$ & $\begin{array}{c}\text { OS (months) } \\
\text { Odds ratio (95\% CI) }\end{array}$ & - \\
\hline Tumor size $(0:<5 \mathrm{~cm}, 1: \geq 5 \mathrm{~cm})$ & $2.213(1.188-4.122)$ & 0.012 & 0.632 \\
Tumor thrombus $(0=$ no, $1=$ yes $)$ & $2.385(1.224-4.647)$ & 0.011 & $2.302(1.173-4.517)$ \\
UCSF criteria $(0=$ no, $1=$ yes $)$ & - & 0.471 & $2.209(1.181-4.131)$ \\
Recipient IL-15 rs10519613 genotype & & & 0.015 \\
$(0=$ CC, $1=$ CA/AA) & $2.214(1.041-4.708)$ & 0.039 & $3.152(1.358-7.315)$ \\
\hline
\end{tabular}

DFS: disease-free survival; OS: overall survival; CI: confidence interval.

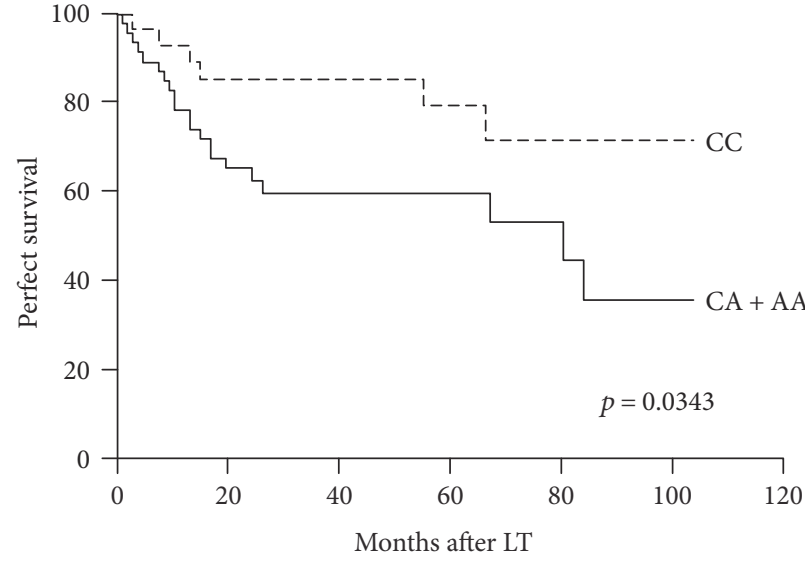

(a)

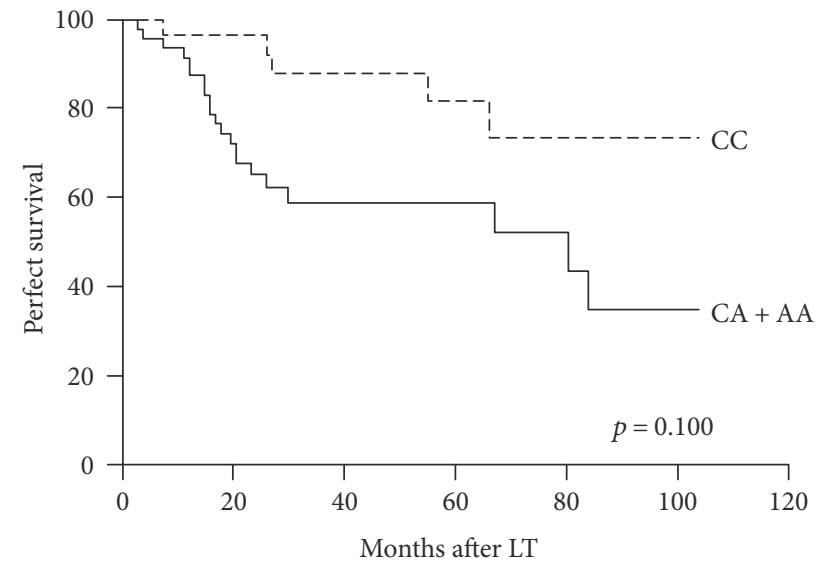

(b)

FIgURE 2: Disease-free survival (a) and overall survival (b) of transplant HCC patients within the UCSF criteria stratified by recipient IL-15 rs10519613 genotypes.

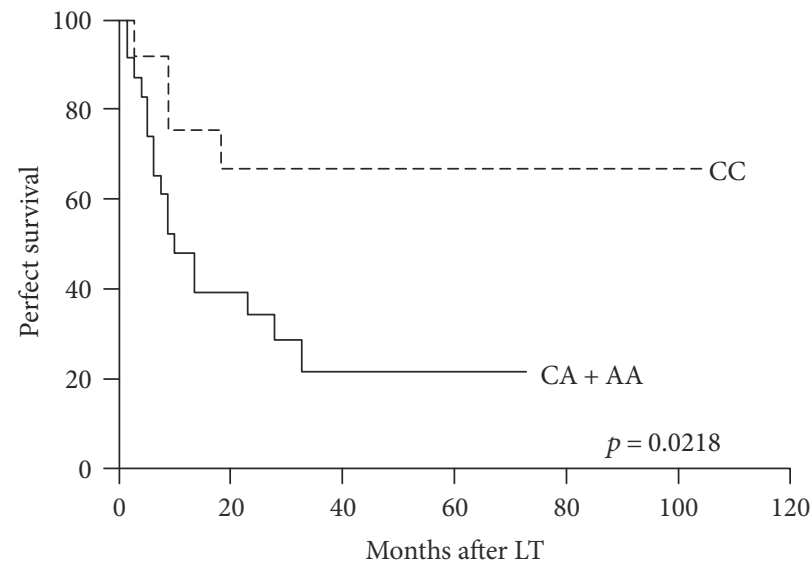

(a)

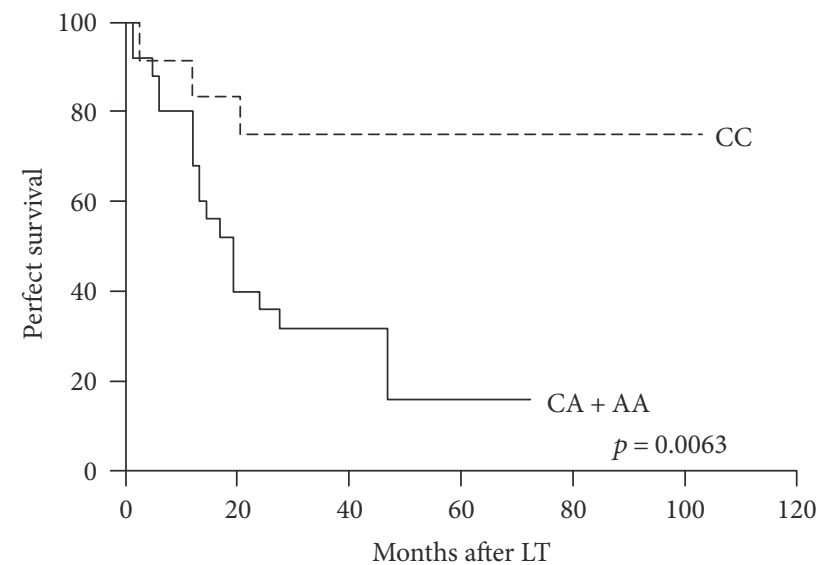

(b)

Figure 3: Disease-free survival (a) and overall survival (b) of transplant HCC patients beyond the UCSF criteria stratified by recipient IL-15 rs10519613 genotypes.

A close relationship between cytokine gene polymorphisms and HCC and HCC prognosis has been reported in recent years $[10,20,21]$. The potential association between the IL15 gene polymorphisms and HCC recurrence and prognosis has been described in several studies. A recent study has shown that IL-15 protein levels in peritumoral liver tissues are significantly associated with a decreased incidence of HCC recurrence and a prolonged overall survival [17].
Furthermore, the genetic constitution is related to the level and function of cytokines and thus affects the antitumor immunity of the host. Several reports have demonstrated that IL-15 gene polymorphisms were significantly related to cancer risk and overall survival $[32,33]$. Moreover, the A allele of IL-15 rs10519613 has been identified as a risk allele for MRD-positive status that is the most prognostic value for risk assessment in ALL [34]. 
In addition to the genetic factor, clinical parameters (tumor thrombus, UCSF criteria) were other independent factors influencing the clinical outcome of HCC patients after LT, which were consistent with the previous studies $[3,35-$ 37]. When combining the genetic factor (recipient IL-15 rs10519613 polymorphism) and UCSF criteria to predict the clinical outcome after LT, we found that recipient IL-15 rs10519613 genetic variant was able to stratify transplant patients within or beyond the UCSF criteria into more homogenous groups with distinct DFS and OS after LT. These results suggested that recipient IL-15 rs10519613 polymorphism could discriminate the clinical outcome of HCC patients within or beyond the UCSF criteria and might affect follow-up and therapeutic strategies after LT.

There were several limitations in our study. Firstly, these results were obtained from a relatively small number of Chinese patients. Therefore, larger sample size is warranted to confirm our findings. Secondly, the biological function of IL-15 rs 10519613 polymorphism was not performed in this study. Assessment of the functionality of this SNP associated with the clinical outcome of HCC after LT is needed in further studies.

In summary, our results demonstrated that recipient IL-15 rs 10519613 genetic variant was an independent predictive factor of HCC recurrence after LT, and integration of this novel genetic marker into the UCSF criteria was helpful for improving the accuracy of prognostication. Therefore, these findings might help to tailor postoperative management strategies for patients with a higher risk of HCC recurrence after LT.

\section{Abbreviations}

LT: $\quad$ Liver transplantation

IL-15: Interleukin 15

HCC: Hepatocellular carcinoma

AFP: Alpha-fetoprotein

NK cells: Natural killer cells

HBV: Hepatitis B virus

DFS: Disease-free survival

OS: Overall survival

SNP: $\quad$ Single-nucleotide polymorphism

MRD: $\quad$ Minimal residual disease

ALL: $\quad$ Acute lymphoblastic leukemia.

\section{Conflicts of Interest}

All of the authors declare they have no competing interests.

\section{Authors' Contributions}

Tao Zhang and Yuan Liu conducted the studies, participated in the collection of data, and drafted the manuscript. Junwei Fan and Zhihai Peng performed the statistical analysis and participated in its design. Xu Peng helped to draft the manuscript. All of the authors read and approved the final manuscript. Tao Zhang, Yuan Liu, and Xu Peng are co-first authors with the same contribution to the paper.

\section{Acknowledgments}

This work was supported by the National Natural Science Foundation of China (Grant nos. 81530044, 81202609, and 8140301).

\section{References}

[1] L. A. Torre, F. Bray, R. L. Siegel, J. Ferlay, J. Lortet-Tieulent, and A. Jemal, "Global cancer statistics, 2012," CA: a Cancer Journal for Clinicians, vol. 65, no. 2, pp. 87-108, 2015.

[2] H. B. El-Serag, "Hepatocellular carcinoma," New England Journal of Medicine, vol. 365, no. 12, pp. 1118-1127, 2011.

[3] F. Y. Yao, L. Xiao, N. M. Bass, R. Kerlan, N. L. Ascher, and J. P. Roberts, "Liver transplantation for hepatocellular carcinoma: validation of the UCSF-expanded criteria based on preoperative imaging," American Journal of Transplantation, vol. 7, no. 11, pp. 2587-2596, 2007.

[4] V. Mazzaferro, J. M. Llovet, R. Miceli et al., "Predicting survival after liver transplantation in patients with hepatocellular carcinoma beyond the Milan criteria: a retrospective, exploratory analysis," The Lancet Oncology, vol. 10, no. 1, pp. 35-43, 2009.

[5] J. P. Duffy, A. Vardanian, E. Benjamin et al., "Liver transplantation criteria for hepatocellular carcinoma should be expanded: a 22-year experience with 467 patients at UCLA," Annals of Surgery, vol. 246, no. 3, pp. 502-511, 2007.

[6] C. Duvoux, F. Roudot-Thoraval, T. Decaens et al., "Liver transplantation for hepatocellular carcinoma: a model including $\alpha$-fetoprotein improves the performance of Milan criteria," Gastroenterology, vol. 143, no. 4, pp. 986-994.e3, 2012.

[7] B. Hameed, N. Mehta, G. Sapisochin, J. P. Roberts, and F. Y. Yao, "Alpha-fetoprotein level $>1000 \mathrm{ng} / \mathrm{mL}$ as an exclusion criterion for liver transplantation in patients with hepatocellular carcinoma meeting the Milan criteria," Liver Transplantation, vol. 20, no. 8, pp. 945-951, 2014.

[8] A. R. Hakeem, R. S. Young, G. Marangoni, J. P. Lodge, and K. R. Prasad, "Systematic review: the prognostic role of alpha-fetoprotein following liver transplantation for hepatocellular carcinoma," Alimentary Pharmacology \& Therapeutics, vol. 35, no. 9, pp. 987-999, 2012.

[9] J. Bruix and J. M. Llovet, "Major achievements in hepatocellular carcinoma," The Lancet, vol. 373, no. 9664, pp. 614-616, 2009.

[10] D. Chen, S. Liu, S. Chen et al., "Donor interleukin 6 gene polymorphisms predict the recurrence of hepatocellular carcinoma after liver transplantation," International Journal of Clinical Oncology, vol. 21, no. 6, pp. 1111-1119, 2016.

[11] A. Fuchs, W. Vermi, J. S. Lee et al., "Intraepithelial type 1 innate lymphoid cells are a unique subset of IL-12- and IL15-responsive IFN- $\gamma$-producing cells," Immunity, vol. 38, no. 4, pp. 769-781, 2013.

[12] S. Dubois, J. Mariner, T. A. Waldmann, and Y. Tagaya, "IL$15 \mathrm{R} \alpha$ recycles and presents IL-15 in trans to neighboring cells," Immunity, vol. 17, no. 5, pp. 537-547, 2002.

[13] J. Zhang, R. Sun, H. Wei, and Z. Tian, "Characterization of interleukin-15 gene-modified human natural killer cells: implications for adoptive cellular immunotherapy," Haematologica, vol. 89, no. 3, pp. 338-347, 2004.

[14] T. A. Waldmann, "The biology of interleukin-2 and interleukin-15: implications for cancer therapy and vaccine design," Nature Reviews Immunology, vol. 6, no. 8, pp. 595-601, 2006. 
[15] W. Jiang, J. Zhang, and Z. Tian, "Functional characterization of interleukin-15 gene transduction into the human natural killer cell line NKL," Cytotherapy, vol. 10, no. 3, pp. 265-274, 2008.

[16] T. A. Waldmann, "Interleukin-15 in the treatment of cancer," Expert Review of Clinical Immunology, vol. 10, no. 12, pp. 1689-1701, 2014.

[17] H. Zhou, H. Huang, J. Shi et al., "Prognostic value of interleukin 2 and interleukin 15 in peritumoral hepatic tissues for patients with hepatitis B-related hepatocellular carcinoma after curative resection," Gut, vol. 59, no. 12, pp. 1699-1708, 2010.

[18] D. L. Blum, T. Koyama, A. E. M'Koma et al., "Chemokine markers predict biochemical recurrence of prostate cancer following prostatectomy," Clinical Cancer Research, vol. 14, no. 23, pp. 7790-7797, 2008.

[19] A. Budhu, M. Forgues, Q. H. Ye et al., "Prediction of venous metastases, recurrence, and prognosis in hepatocellular carcinoma based on a unique immune response signature of the liver microenvironment," Cancer Cell, vol. 10, no. 2, pp. 99-111, 2006.

[20] Y. Wang, N. Kato, Y. Hoshida et al., "Interleukin- $1 \beta$ gene polymorphisms associated with hepatocellular carcinoma in hepatitis C virus infection," Hepatology, vol. 37, no. 1, pp. 65-71, 2003.

[21] K. Migita, S. Miyazoe, Y. Maeda et al., "Cytokine gene polymorphisms in Japanese patients with hepatitis B virus infectio$\mathrm{n}$-association between TGF- $\beta 1$ polymorphisms and hepatocellular carcinoma," Journal of Hepatology, vol. 42, no. 4, pp. 505-510, 2005.

[22] H. Sun, M. Teng, J. Liu et al., "FOXM1 expression predicts the prognosis in hepatocellular carcinoma patients after orthotopic liver transplantation combined with the Milan criteria," Cancer Letters, vol. 306, no. 2, pp. 214-222, 2011.

[23] F. Y. Yao, L. Ferrell, N. M. Bass et al., "Liver transplantation for hepatocellular carcinoma: expansion of the tumor size limits does not adversely impact survival," Hepatology, vol. 33, no. 6, pp. 1394-1403, 2001.

[24] S. Gabriel, L. Ziaugra, and D. Tabbaa, "SNP genotyping using the Sequenom MassARRAY iPLEX platform," Current Protocols in Human Genetics, vol. 60, pp. 2.12.1-2.12.18, 2009.

[25] Y. Y. Shi and L. He, "SHEsis, a powerful software platform for analyses of linkage disequilibrium, haplotype construction, and genetic association at polymorphism loci," Cell Research, vol. 15, no. 2, pp. 97-98, 2005.

[26] J. K. Yamamoto-Furusho, J. L. De-Leon-Rendon, E. AlvarezLeon, M. Valdivia-Eziquio, C. Obil-Chavarria, and G. VargasAlarcon, "Association of the interleukin 15 (IL-15) gene polymorphisms with the risk of developing ulcerative colitis in Mexican individuals," Molecular Biology Reports, vol. 41, no. 4, pp. 2171-2176, 2014.

[27] Z. Wu and Y. Xu, "IL-15R $\alpha$-IgG1-Fc enhances IL-2 and IL-15 anti-tumor action through $\mathrm{NK}$ and $\mathrm{CD}^{+} \mathrm{T}$ cells proliferation and activation," Journal of Molecular Cell Biology, vol. 2, no. 4, pp. 217-222, 2010.

[28] D. Liu, L. Song, J. Wei et al., "IL-15 protects NKT cells from inhibition by tumor-associated macrophages and enhances antimetastatic activity," The Journal of Clinical Investigation, vol. 122, no. 6, pp. 2221-2233, 2012.

[29] K. B. Stephenson, N. G. Barra, E. Davies, A. A. Ashkar, and B. D. Lichty, "Expressing human interleukin-15 from oncolytic vesicular stomatitis virus improves survival in a murine metastatic colon adenocarcinoma model through the enhancement of anti-tumor immunity," Cancer Gene Therapy, vol. 19, no. 4, pp. 238-246, 2012.

[30] J. C. Morris, C. A. Ramlogan-Steel, P. Yu et al., "Vaccination with tumor cells expressing IL-15 and IL-15R $\alpha$ inhibits murine breast and prostate cancer," Gene Therapy, vol. 21, no. 4, pp. 393-401, 2014.

[31] W. Jiang, C. Zhang, Z. Tian, and J. Zhang, "hIL-15 genemodified human natural killer cells (NKL-IL15) augments the anti-human hepatocellular carcinoma effect in vivo," Immunobiology, vol. 219, no. 7, pp. 547-553, 2014.

[32] K. L. Bondurant, A. Lundgreen, J. S. Herrick, S. Kadlubar, R. K. Wolff, and M. L. Slattery, "Interleukin genes and associations with colon and rectal cancer risk and overall survival," International Journal of Cancer, vol. 132, no. 4, pp. 905-915, 2013.

[33] A. L. Van Dyke, M. L. Cote, A. S. Wenzlaff et al., "Cytokine and cytokine receptor single-nucleotide polymorphisms predict risk for non-small cell lung cancer among women," Cancer Epidemiology, Biomarkers \& Prevention, vol. 18, no. 6, pp. 1829-1840, 2009.

[34] M. Dawidowska, M. Kosmalska, Ł. Sędek et al., "Association of germline genetic variants in RFC, IL15 and VDR genes with minimal residual disease in pediatric B-cell precursor ALL," Scientific Reports, vol. 6, 2016.

[35] Y. P. Fu, Y. Yi, J. L. Huang et al., "Prognostic nomograms stratify survival of patients with hepatocellular carcinoma without portal vein tumor thrombosis after curative resection," The Oncologist, vol. 22, no. 5, pp. 561-569, 2017.

[36] H. J. Choi, D. G. Kim, G. H. Na et al., "The clinical outcomes of patients with portal vein tumor thrombi after living-donor liver transplantation," Liver Transplantation, vol. 23, no. 8, pp. 1023-1031, 2017.

[37] V. G. Agopian, M. M. Morshedi, J. McWilliams et al., "Complete pathologic response to pretransplant locoregional therapy for hepatocellular carcinoma defines cancer cure after liver transplantation: analysis of 501 consecutively treated patients," Annals of Surgery, vol. 262, no. 3, pp. 536-545, 2015. 


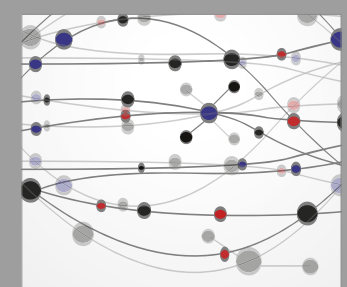

The Scientific World Journal
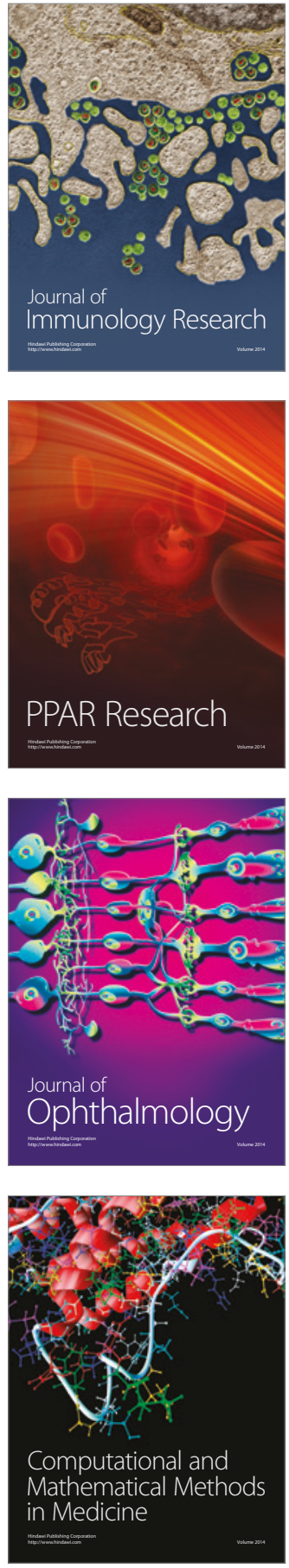

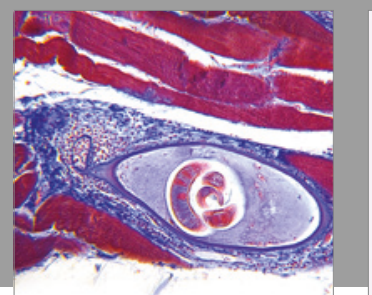

Gastroenterology Research and Practice
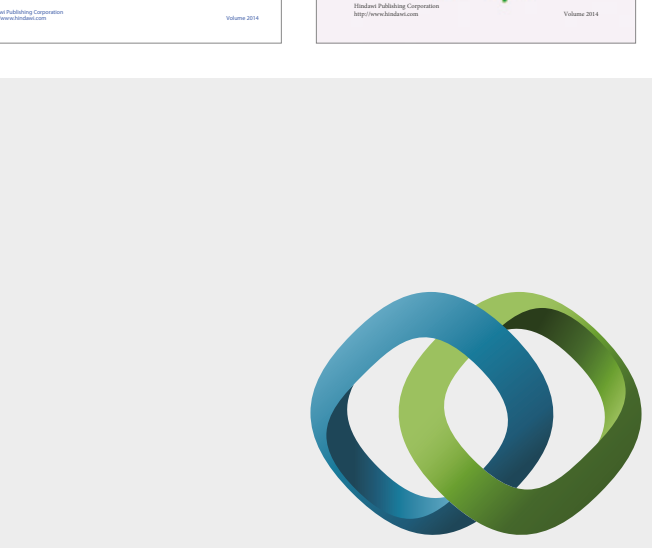

\section{Hindawi}

Submit your manuscripts at

https://www.hindawi.com
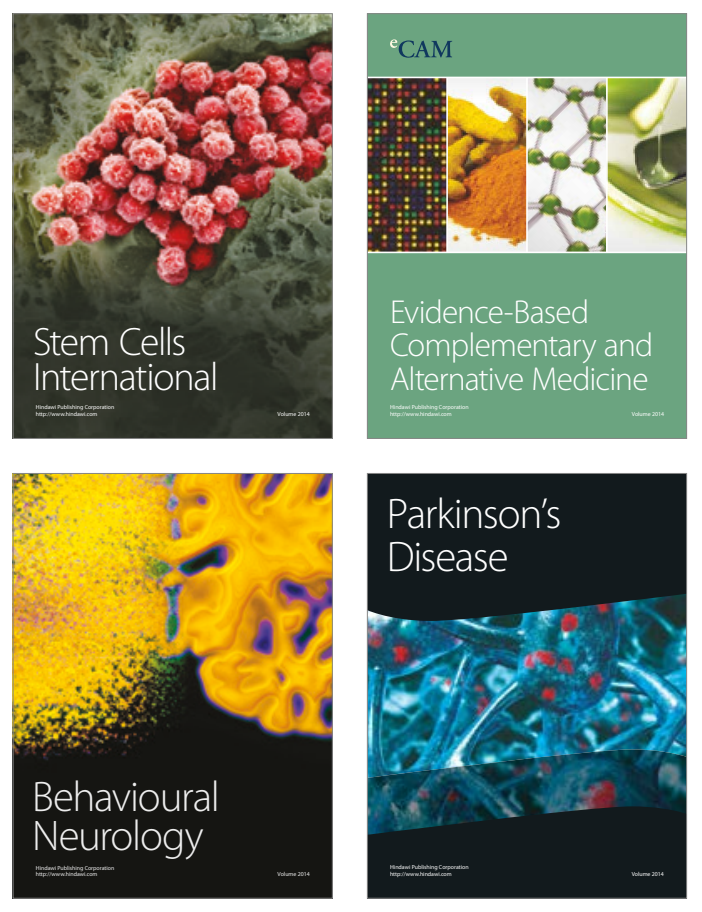
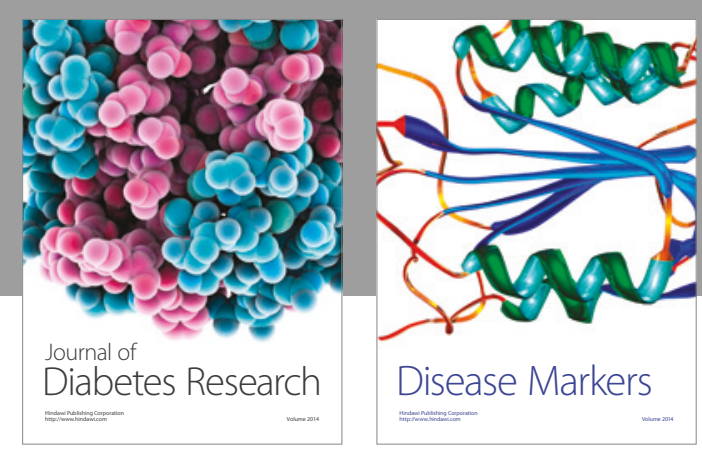

Disease Markers
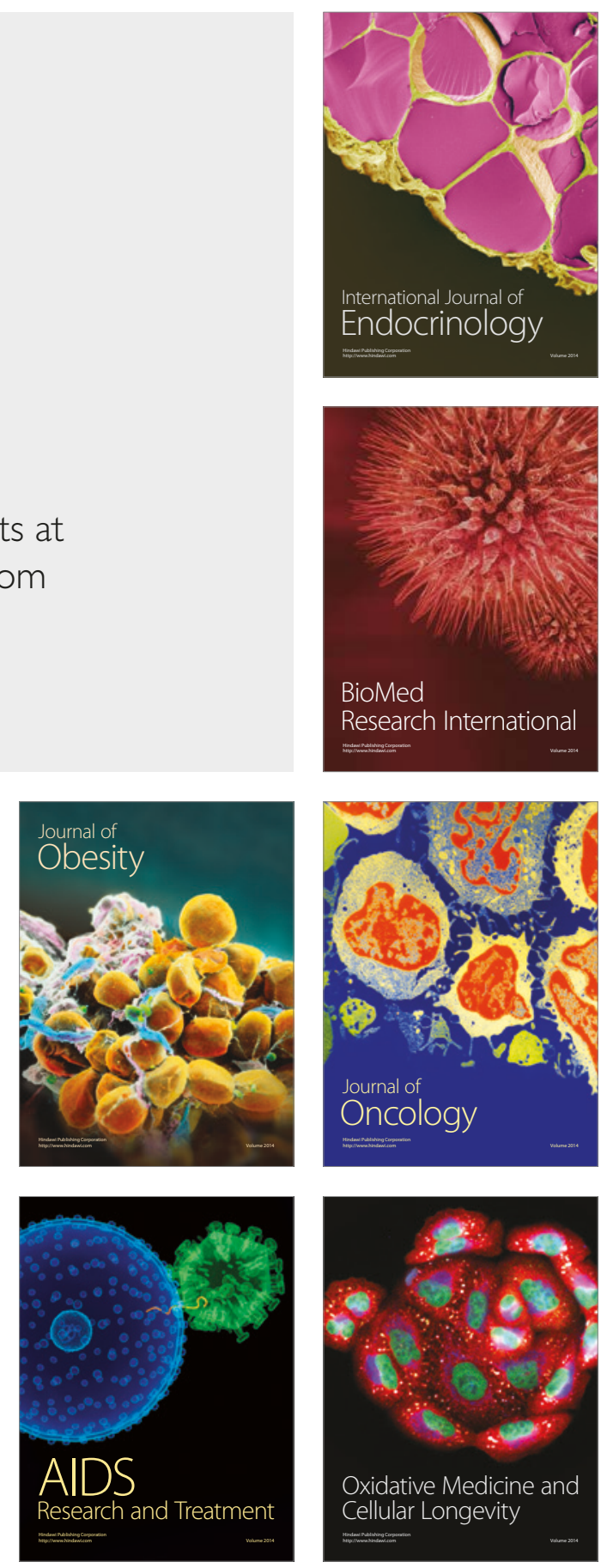